\title{
Antiarrhythmic medication is superior to catheter ablation in suppressing supraventricular ectopic complexes in patients with atrial fibrillation
}

\section{Alhede, Christina}

2017-10-01

Alhede , C , Lauridsen , T K, Johannessen, A, Dixen, U , Jensen , J S , Raatikainen , P , Hindricks, G, Walfridsson, H, Kongstad, O , Pehrson , S, Englund, A, Hartikainen , J , Hansen, P S, Nielsen, J C \& Jons , C 2017 , ' Antiarrhythmic medication is superior to catheter ablation in suppressing supraventricular ectopic complexes in patients with atrial fibrillation ' , International Journal of Cardiology , vol. 244 , pp. 186-191 . https://doi.org/10.1016/j.ijcard.2017.05.028

http://hdl.handle.net/10138/297901

https://doi.org/10.1016/j.ijcard.2017.05.028

unspecified

publishedVersion

Downloaded from Helda, University of Helsinki institutional repository.

This is an electronic reprint of the original article.

This reprint may differ from the original in pagination and typographic detail.

Please cite the original version. 


\title{
Antiarrhythmic medication is superior to catheter ablation in suppressing supraventricular ectopic complexes in patients with atrial fibrillation
}

\author{
Christina Alhede ${ }^{\mathrm{a}, *, 1}$, Trine K. Lauridsen ${ }^{\mathrm{a}, 1}$, Arne Johannessen ${ }^{\mathrm{a}, 1}$, Ulrik Dixen ${ }^{\mathrm{b}, 1}$, Jan S. Jensen ${ }^{\mathrm{a}, 1}$, \\ Pekka Raatikainen c,1 $^{\text {, Gerhard Hindricks d,1, Haakan Walfridsson e,1 }}{ }^{\text {e, Ole Kongstad }}{ }^{\text {f,1 }}$, Steen Pehrson ${ }^{\text {g,1, }}$ \\ Anders Englund $^{\text {h,1 }}$, Juha Hartikainen ${ }^{\mathrm{i}, 1}$, Peter S. Hansen ${ }^{\mathrm{j}, 1}$, Jens C. Nielsen ${ }^{\mathrm{k}, 1}$, Christian Jons ${ }^{\mathrm{g}, 1}$ \\ a Herlev-Gentofte University Hospital, Denmark \\ b Hvidovre University Hospital, Hvidovre, Denmark \\ c Helsinki University Hospital, Helsinki, Finland \\ d Leipzig University Hospital, Leipzig, Germany \\ e Linkoping University Hospital, Linkoping, Sweden \\ ${ }^{\mathrm{f}}$ Lund University Hospital, Lund, Sweden \\ g Rigshospitalet, Copenhagen, Denmark \\ ${ }^{\text {h }}$ Orebro University Hospital, Orebro, Sweden \\ ${ }^{\mathrm{i}}$ Kupio University Hospital, Kupio, Finland \\ j Varde Heart Center, Varde, Denmark \\ ${ }^{\mathrm{k}}$ Aarhus University Hospital, Aarhus, Denmark
}

\section{A R T I C L E I N F O}

\section{Article history:}

Received 8 March 2017

Received in revised form 5 May 2017

Accepted 8 May 2017

Available online 10 May 2017

Keywords:

Arrhythmia

Treatment

Ablation

Premature ectopic beats

Recurrence

\begin{abstract}
A B S T R A C T
Background: Supraventricular ectopic complexes (SVEC) originating in the pulmonary veins are known triggers of atrial fibrillation (AF) which led to the development of pulmonary vein isolation for AF. However, the long-term prevalence of SVEC after catheter ablation (CA) as compared to antiarrhythmic medication (AAD) is unknown. Our aims were to compare the prevalence of SVEC after AAD and CA and to estimate the association between baseline SVEC burden and AF burden during 24 months of follow-up.

Methods: Patients with paroxysmal AF $(N=260)$ enrolled in the MANTRA PAF trial were treated with AAD $(N=$ 132 ) or CA $(N=128)$. At baseline and 3, 6, 12, 18 and 24 months follow-up patients underwent 7-day Holter monitoring to assess SVEC and AF burden. We compared SVEC burden between treatments with Wilcoxon sum rank test. Results: Patients treated with AAD had significantly lower daily SVEC burden during follow-up as compared to CA (AAD: 19 [6-58] versus CA: 39 [14-125], $p=0.003$ ). SVEC burden increased post-procedurally followed by a decrease after CA whereas after AAD SVEC burden decreased and stabilized after 3 months of follow-up. Patients with low SVEC burden had low AF burden after both treatments albeit this was more pronounced after CA at 24 months of follow-up.

Conclusion: AAD was superior to CA in suppressing SVEC burden after treatment of paroxysmal AF. After CA SVEC burden increased immediately post-procedural followed by a decrease whereas after AAD an early decrease was observed. Lower SVEC burden was highly associated with lower AF burden during follow-up especially after CA.
\end{abstract}

(c) 2017 Elsevier B.V. All rights reserved.

\section{Introduction}

Today, first-line treatment of paroxysmal AF is antiarrhythmic medication or catheter ablation in selected patients [1]. However, challenges

\footnotetext{
* Corresponding author at: Cardiovascular Research II, Herlev-Gentofte University Hospital, Kildegaardsvej 28, 2900 Hellerup, Denmark.

E-mail address: christina.alhede@regionh.dk (C. Alhede).

1 This author takes responsibility for all aspects of the reliability and freedom from bias of the data presented and their discussed interpretation.
}

remain for both treatment methods; antiarrhythmic medication can be associated with severe adverse effects and the recurrence rate after catheter ablation remains high [1-3].

Numerous studies have demonstrated that ectopic complexes with short coupling interval are the primary trigger of AF paroxysms [4-8]. In the landmark study by Haïssaguerre et al., it was discovered that $94 \%$ of ectopic complexes initiating AF originated from the pulmonary veins which led to the current state-of-the-art catheter ablation strategy with pulmonary vein isolation [4]. However, patients with paroxysmal AF often experience frequent palpitations after catheter ablation 
which can be associated with reduced quality of life. Furthermore, our group and others have demonstrated that high prevalence of SVEC is highly associated with AF recurrence after catheter ablation [9-11] indicating that SVEC may be an important trigger of recurrent AF.

Furthermore, little knowledge exists about the prevalence of SVEC after treatment with antiarrhythmic medication. Kirchhof et al. demonstrated that flecainide prolongs the atrial post-repolarization refractoriness which hypothetically could suppress the prevalence of SVEC during treatment with flecainide [12]. However, it is unclear whether antiarrhythmic medication as compared to catheter ablation suppresses the prevalence of SVEC during long-term follow-up and whether SVEC burden is associated with long-term AF burden. Improving our knowledge of long-term SVEC burden may contribute to identify possible underlying mechanisms responsible for $\mathrm{AF}$ recurrence after treatment and add important prognostic evidence following treatment with antiarrhythmic medication or catheter ablation of paroxysmal AF. Thus, our aim was to investigate the long-term prevalence of SVEC after antiarrhythmic medication and catheter ablation, respectively and the association between baseline SVEC burden and long-term AF burden in patients with paroxysmal AF initially randomized to first-line treatment with antiarrhythmic medication or catheter ablation.

\section{Methods}

\subsection{Population}

The patients included in this study were enrolled in a randomized clinical trial The Medical Antiarrhythmic Treatment or Radiofrequency Ablation in Paroxysmal Atrial Fibrillation (MANTRA PAF) comparing antiarrhythmic medication and catheter ablation as first-line treatment of paroxysmal AF [13,14] (ClinicalTrials.gov number, NCT00133211). The study included 294 patients with symptomatic paroxysmal AF. Exclusion criteria were age $>70$ years, left atrial size $>50 \mathrm{~mm}$, left ventricular ejection fraction (LVEF) $<40 \%$ and structural or valvular heart disease. The study was conducted according to the Helsinki Declaration and approved by regional ethics committees. All patients gave written consent before enrolment. No sex-based differences were present.

The patients were initially randomized to treatment with either antiarrhythmic medication or catheter ablation. In the current study patients who did not receive the initial assigned treatment or who did not undergo 7-day Holter monitoring at baseline were excluded, and a total of 260 patients were included in the study with 132 patients randomized and treated with antiarrhythmic medication and 128 patients undergoing catheter ablation.

\subsection{Catheter ablation and antiarrhythmic medication}

Wide antral ablation with encircling of ipsilateral veins combined with a posterior line was mandatory [14]. Additional ablation lines were on the operator's discretion. The procedural endpoint was absence of high-frequency electrical activity $(>0.2 \mathrm{mV})$ inside the encircled areas around the pulmonary veins. Antiarrhythmic medication was allowed during the blanking period of 3 months after the procedure, and $22(17 \%)$ patients had amiodarone and 25 (20\%) patients had flecainide within the blanking period. After the blanking period antiarrhythmic medication was discouraged. Patients in the catheter ablation group with symptomatic AF recurrence were offered re-ablation with re-pulmonary vein isolation and/or additional linear lines when clinically indicated $(n=53(41 \%)$ ).

First-line antiarrhythmic medication was flecainide at a dose of $200 \mathrm{mg}$ per day or propafenone at a dose of $600 \mathrm{mg}$ per day. In patients with contraindications for class IC antiarrhythmic drugs amiodarone at a dose of $200 \mathrm{mg}$ per day or sotalol at a dose of $160 \mathrm{mg}$ per day were used. In total, 123 (93\%) were treated with flecainide, 4 (3\%) with amiodarone, 2 (2\%) with propafenone and $3(2 \%)$ had sotalol as initial treatment. A $\beta$-blocker, calcium-channel blocker or digoxin was given supplementary to class IC agents according to institutional standards at the involved centers. Details of the recommended dosing have previously been reported [14].

\subsection{Holter monitoring}

Patients underwent 7-day Holter monitoring at baseline and after 3, 6, 12, 18, and 24 months follow-up. Patients randomized to catheter ablation underwent an additional 7-day Holter monitoring two days after the ablation. All Holter recordings were analysed at Aarhus University Hospital by the same experienced technician blinded with respect to randomization. All Holter recordings were scanned using Sentinel Pathfinder Digital (Spacelabs Healthcare) and interpreted using the interactive method. All episodes of AF and SVECs were recorded and summed during the entire recording period. SVEC were defined as supraventricular complexes occurring $>30 \%$ earlier than expected compared to previous R-R interval.

In the present analysis, patients randomized to and treated with antiarrhythmic medication undergoing supplementary catheter ablation during follow-up as well as patients randomized to and treated with catheter ablation receiving supplementary antiarrhythmic medication during follow-up were censored at the time of non-adherence to the randomized treatment.

Patients with $100 \%$ AF during Holter monitoring, who per definition do not have any supraventricular ectopics, were censored. No patients had AF $100 \%$ of the time during Holter monitoring at baseline. Patients with AF burden of $<100 \%$ were included in the study. To account for AF episodes during Holter monitoring and slight variations in recording time we used median number of SVEC per day in sinus rhythm.

In order to estimate the change in SVEC burden during follow-up according to baseline SVEC burden, patients were allocated according to low, moderate and high SVEC burden defined as 0-25 SVEC/day, 25-100 SVEC/day and >100 SVEC/day. Further, high SVEC burden was defined as SVEC $>100$ /day in correspondence to a report from Varounis et al. [15] and AF burden was defined as the percentage of time in AF on each Holter monitoring.

\subsection{Statistics}

The long-term prevalence of SVEC after treatment with antiarrhythmic medication and catheter ablation of patients with paroxysmal AF was assessed. Continuous variables were presented as medians with 25 th and 75 th percentiles. Categorical variables were presented as frequencies and percentages of the specified group. Two-tailed $P$ values $<$ 0.05 were pre-specified to indicate statistical significance.

Comparisons of baseline characteristics according to SVEC burden $>100$ SVEC/day were made with Student's $t$-test for continuous variables and with $\chi^{2}$ test or Fisher's exact test for categorical variables. The distribution of continuous variables was assessed using visual inspection of the histogram.

Wilcoxon rank sum test was used to compare the difference in SVEC burden at follow-up visits between patients treated with antiarrhythmic medication and catheter ablation and to compare the changes in SVEC burden during follow-up as well as to compare median AF burden between patients with low, moderate and high SVEC burden at the same 7-day Holter monitoring during follow-up. Finally, in order to investigate SVEC burden and other patient characteristics associated with presence of $\mathrm{AF}$ on Holter monitoring we defined $\mathrm{AF}>0$ on Holter monitoring at baseline and after 24 months of follow-up as endpoint and univariate and multivariate logistic regression models were used. Patient characteristics entered into the model were considered to be variables of interest or confounders and patient characteristics with a $p<0.10$ remained in the final multivariate model. All analyses were performed using SAS version 9.4 (SAS Institute Inc., Cary, NC, US).

\section{Results}

\subsection{Study population}

Baseline characteristics stratified by SVEC burden during baseline Holter monitoring are shown in Table 1. Patients with SVEC burden $>$ 100 /day were older, fewer patients had previous cardioversion, and they had more frequent AF episodes. Patients with $>100$ SVEC/day at baseline were equally distributed between patients treated with antiarrhythmic medication and catheter ablation, respectively.

\subsection{Long-term SVEC burden}

The long-term prevalence of SVEC per day from 7-day Holter monitoring at baseline and during 24 months of follow-up according to treatment is shown in Fig. 1. At baseline, SVEC burden was equally distributed in the two treatment groups $(p=0.8)$. In the catheter ablation group, an increase in the prevalence of SVEC was seen immediately after the ablation procedure. After 3 months, the SVEC burden decreased in patients treated with catheter ablation. Hereafter, SVEC burden stabilized through 24 months of follow-up. Patients treated with antiarrhythmic medication had a significantly lower prevalence of SVEC during follow-up compared to patients treated with catheter ablation throughout follow-up $(p=0.003)$. Furthermore, patients treated with antiarrhythmic medication showed a different pattern with an initial decrease followed by stabilization in SVEC burden 3 months after treatment initiation.

\subsection{Change in SVEC burden}

The average reduction in SVEC burden from baseline was 73\% in patients treated with antiarrhythmic medication and 52\% after catheter ablation at 24 months follow-up. In the antiarrhythmic medication 
Table 1

Patient characteristics stratified by prevalence of supraventricular ectopic complexes per day at baseline.

\begin{tabular}{|c|c|c|c|}
\hline & SVEC $\leq 100$ & SVEC $>100$ & \\
\hline & $N=141$ & $N=119$ & $P$ \\
\hline \multicolumn{4}{|l|}{ Characteristics } \\
\hline Age, y & $54(10)$ & $57(8)$ & $<0.006$ \\
\hline Female sex, n (\%) & $40(28)$ & $34(29)$ & 1.00 \\
\hline Body Mass Index, $\mathrm{kg} / \mathrm{m}^{2}$ & $27(4)$ & $26(3)$ & 0.04 \\
\hline \multicolumn{4}{|l|}{ AF history } \\
\hline Previous cardioversion, n (\%) & $48(34)$ & $29(24)$ & 0.09 \\
\hline \multicolumn{4}{|l|}{ Interval between $\mathrm{AF}$ episodes } \\
\hline 0-7 days, $\mathrm{n}(\%)$ & $119(84)$ & $110(78)$ & \\
\hline$>7$ days, $\mathrm{n}(\%)$ & $22(16)$ & $8(7)$ & 0.02 \\
\hline \multicolumn{4}{|l|}{ Average duration of AF episodes } \\
\hline$\leq 1 \mathrm{~h}, \mathrm{n}(\%)$ & $78(55)$ & 70 (59) & \\
\hline$>1 \mathrm{~h}$ and $<24 \mathrm{~h}, \mathrm{n}(\%)$ & $50(35)$ & $34(29)$ & \\
\hline$>24 \mathrm{~h}, \mathrm{n}(\%)$ & $11(8)$ & $14(12)$ & 0.13 \\
\hline \multicolumn{4}{|l|}{ Medical history } \\
\hline Hypertension, n (\%) & $48(34)$ & $33(27)$ & 0.34 \\
\hline Coronary artery disease, n (\%) & $4(3)$ & $3(3)$ & 1.00 \\
\hline Valvular Disease, $\mathrm{n}(\%)$ & $6(4)$ & $5(4)$ & 1.00 \\
\hline Pacemaker, n (\%) & $4(3)$ & $7(6)$ & 0.35 \\
\hline Diabetes, n (\%) & $9(6)$ & $3(3)$ & 0.24 \\
\hline Chronic lung disease, n (\%) & $9(6)$ & $3(3)$ & 0.24 \\
\hline Thyroid disease, $\mathrm{n}(\%)$ & $10(7)$ & $5(4)$ & 0.42 \\
\hline \multicolumn{4}{|l|}{ Echocardiography } \\
\hline Left atrial diameter, $\mathrm{mm}$ & $40.1(5.5)$ & $39.8(5.2)$ & 0.66 \\
\hline LV ejection fraction, \% & $65.3(8.1)$ & $63.0(8.9)$ & 0.05 \\
\hline \multicolumn{4}{|l|}{ Medication } \\
\hline$\beta$-Blocker use, n (\%) & $98(70)$ & $87(73)$ & 0.62 \\
\hline Calcium channel blocker use, n (\%) & $20(14)$ & $20(17)$ & 0.68 \\
\hline Digoxin use, n (\%) & $6(4)$ & $16(13)$ & 0.02 \\
\hline \multicolumn{4}{|l|}{ Index treatment } \\
\hline $\mathrm{AAD}, \mathrm{n}(\%)$ & $73(52)$ & $59(50)$ & \\
\hline Catheter Ablation, n (\%) & $68(48)$ & $60(50)$ & 0.72 \\
\hline \multicolumn{4}{|l|}{ Crossover } \\
\hline AAD to catheter ablation, (\%) & $22(16)$ & $34(29)$ & 0.14 \\
\hline Catheter ablation to AAD, $\mathrm{n}(\%)$ & $17(12)$ & $17(14)$ & 0.8 \\
\hline
\end{tabular}

Continuous values are presented as mean (SD). Hr indicates hour, AF; atrial fibrillation, $\mathrm{LV}$ indicates left ventricular; AAD, antiarrhythmic medication; SD, standard deviation.

group, 26 (20\%) patients experienced an increase in SVEC burden whereas this was the case for 46 (36\%) patients in the catheter ablation group $(p=0.07)$.

We further assessed the average change in SVEC burden at 24 months follow-up according to baseline SVEC burden. Fig. 2 illustrates the reduction in the prevalence of SVEC in patients categorized with 0-25 SVEC/day, 25-100 SVEC/day or >100 SVEC/day at baseline after treatment with antiarrhythmic medication (Fig. 2A) and catheter ablation (Fig. 2B). Patients with SVEC burden of 025 SVEC/day experienced an insignificant reduction in SVEC burden after antiarrhythmic medication whereas patients treated with catheter ablation had a significant increase in SVEC burden.

\subsection{AF burden according to SVEC burden}

We further examined the association between AF burden and low, moderate and high SVEC burden measured from the same 7-day Holter monitoring during each follow-up visit in patients treated with antiarrhythmic medication (Fig. 3A) and catheter ablation (Fig. 3B), respectively. At each Holter monitoring, lower SVEC burden was associated with significantly lower AF burden during the same Holter monitoring in patients treated with antiarrhythmic medication. After catheter ablation, patients with lower SVEC burden also had significantly lower AF burden during follow-up than patients with higher SVEC burden. However, at 24 months of follow-up AF burden was very low in all three categories and we could not demonstrate a significant association between SVEC burden and AF burden.

Univariate and multivariate analyses of patient characteristics associated with presence of AF at baseline and 24 months of follow-up for patients treated with antiarrhythmic medication and catheter ablation, respectively, are illustrated in supplemental appendix (Tables 2-5). After multivariate adjustment, high SVEC burden measured at baseline and 24 months of follow-up, respectively, remained significantly associated with presence of AF at baseline (OR 9.0 [3.1-25.9], $p<0.0001$ ) and after 24 months (OR 2.1 [6.4-20.0], $p=0.001$ ) in patients treated with antiarrhythmic medication. After catheter ablation, high baseline SVEC burden were predictive of baseline presence of AF (OR 1.2 [3.4$9.8], p=0.03$ ) but SVEC burden measured at 24 months of follow-up was not associated with presence of AF on the same recording (OR 6.0 [0.6-70], $p=0.2$ ). Finally, we performed sensitivity analyses with and without patients who changed treatment group and found no difference in the results.

\section{Discussion}

\subsection{Major findings}

In this study of patients with paroxysmal AF treated with antiarrhythmic medication or catheter ablation as first-line therapy, we found antiarrhythmic medication superior to catheter ablation in suppressing SVEC. Patients treated with catheter ablation demonstrated a different pattern during follow-up with an increase in SVEC burden immediately post-procedurally followed by a decrease and stabilization at 3 months of follow-up. In comparison, after antiarrhythmic medication an early decrease and stabilization were observed. We further found that lower SVEC burden during follow-up were associated with lower long-term AF burden in both treatment groups albeit this was more pronounced in patients treated with catheter ablation. Our findings add to better understand the presence of supraventricular ectopic triggers after treatment of paroxysmal AF and the results indicate that SVEC burden is an important marker of AF burden after both antiarrhythmic medication and catheter ablation.

\subsection{Long-term SVEC burden}

Presence of non-pulmonary vein triggers may explain the higher SVEC burden in patients treated with catheter ablation. In a study by Takigawa, it was suggested that non-pulmonary vein triggers originating from other foci inside the left atrium are unmasked when the primary pulmonary vein triggers are removed [16]. Catheter ablation with pulmonary vein isolation primarily targets the ectopic complexes originating in the pulmonary veins $[4,5,17,18]$ whereas flecainide-induced atrial post-repolarization refractoriness as demonstrated by Kirchhof et al. targets both pulmonary and nonpulmonary triggers which may explain the lower SVEC burden in patients treated with antiarrhythmic medication [12]. Unfortunately, we could not study the origin of the ectopic complexes as we used a 3-lead set-up for Holter monitoring.

\subsection{Ectopic activity post-procedural}

The marked increase in SVEC burden immediately after catheter ablation may be attributed to acute inflammation caused by atrial tissue damage within the first 3 months after catheter ablation [19,20]. However, as previously demonstrated, because high SVEC burden immediately after catheter ablation predicts long-term AF recurrence it cannot be attributed to acute inflammation alone [11]. In comparison, we found an early decrease in SVEC burden in patients treated with antiarrhythmic medication. However, we could not compare SVEC burden immediately after initiation of antiarrhythmic medication because 


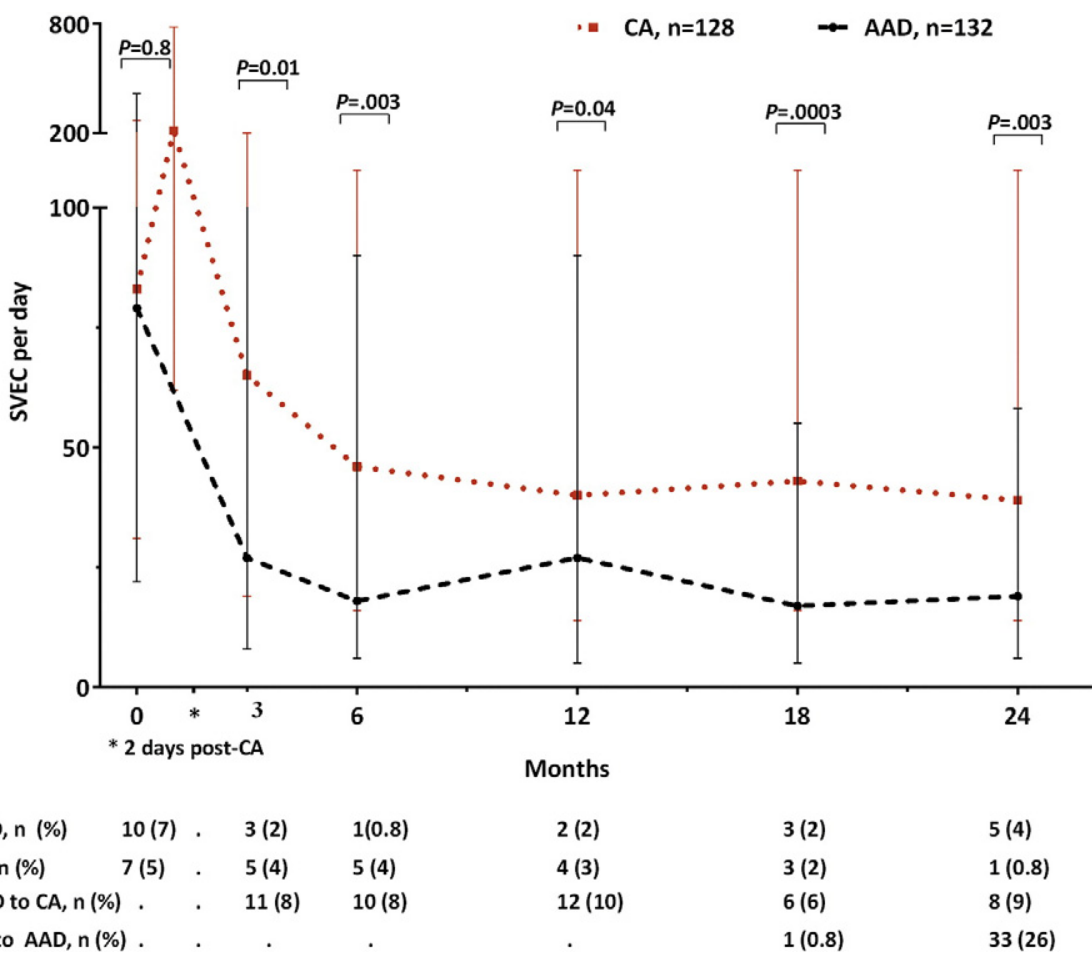

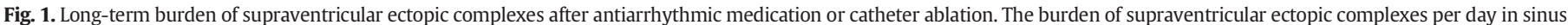

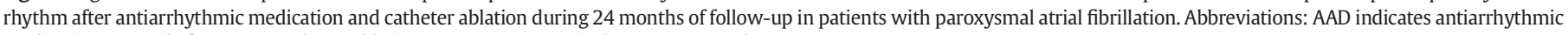
medication; CA, radiofrequency catheter ablation; SVEC, supraventricular ectopic complexes.

patients in the medication group did not undergo Holter monitoring immediately after initiation of treatment.

\subsection{AF burden during follow-up}

We found a lower AF burden in patients with lower SVEC burden during follow-up. This is in line with previous reports that demonstrated an increased risk of AF after catheter ablation with higher SVEC burden [9-11]. In the MANTRA PAF trial there was no difference in AF burden between the two treatments until 24 months where patients treated with catheter ablation had a slightly lower AF burden. This seems contradictive to our results where patients treated with catheter ablation had a higher SVEC burden during follow-up and higher SVEC burden was associated with higher AF burden. Due to the study design, we can only speculate on the cause of these findings. One explanation could be that only some patterns of SVEC may trigger AF depending on timing, prematurity, nature and origin rather than just the frequency of SVEC [21]. Interestingly, in patients treated with catheter ablation AF burden at

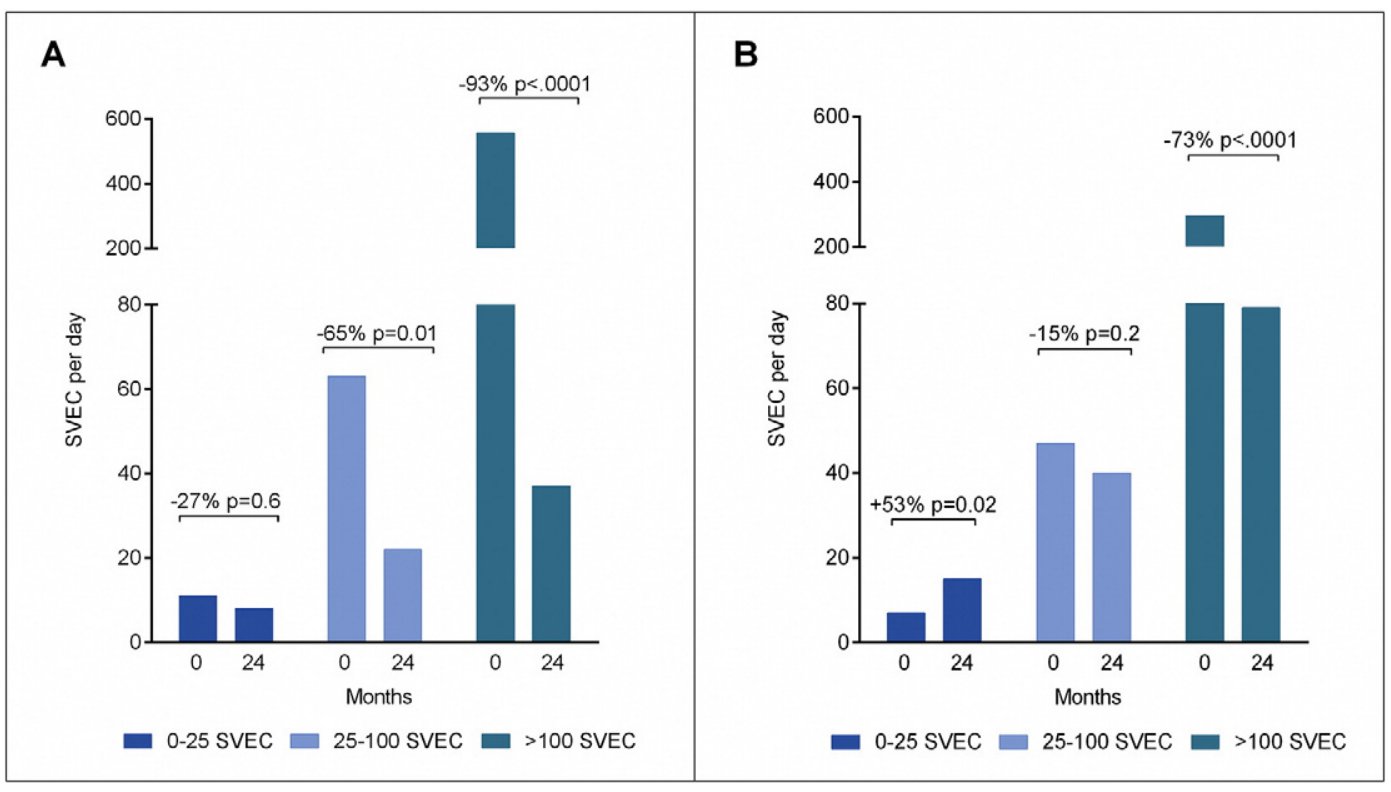

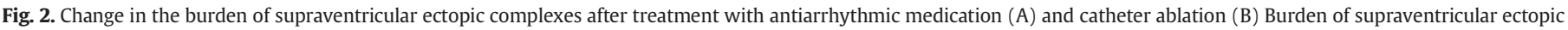

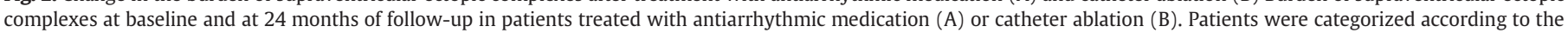
baseline ectopy burden of 0-25 SVEC/day, 25-100 SVEC/day and > 100 SVEC/day. Abbreviations: SVEC indicates supraventricular ectopic complexes. 

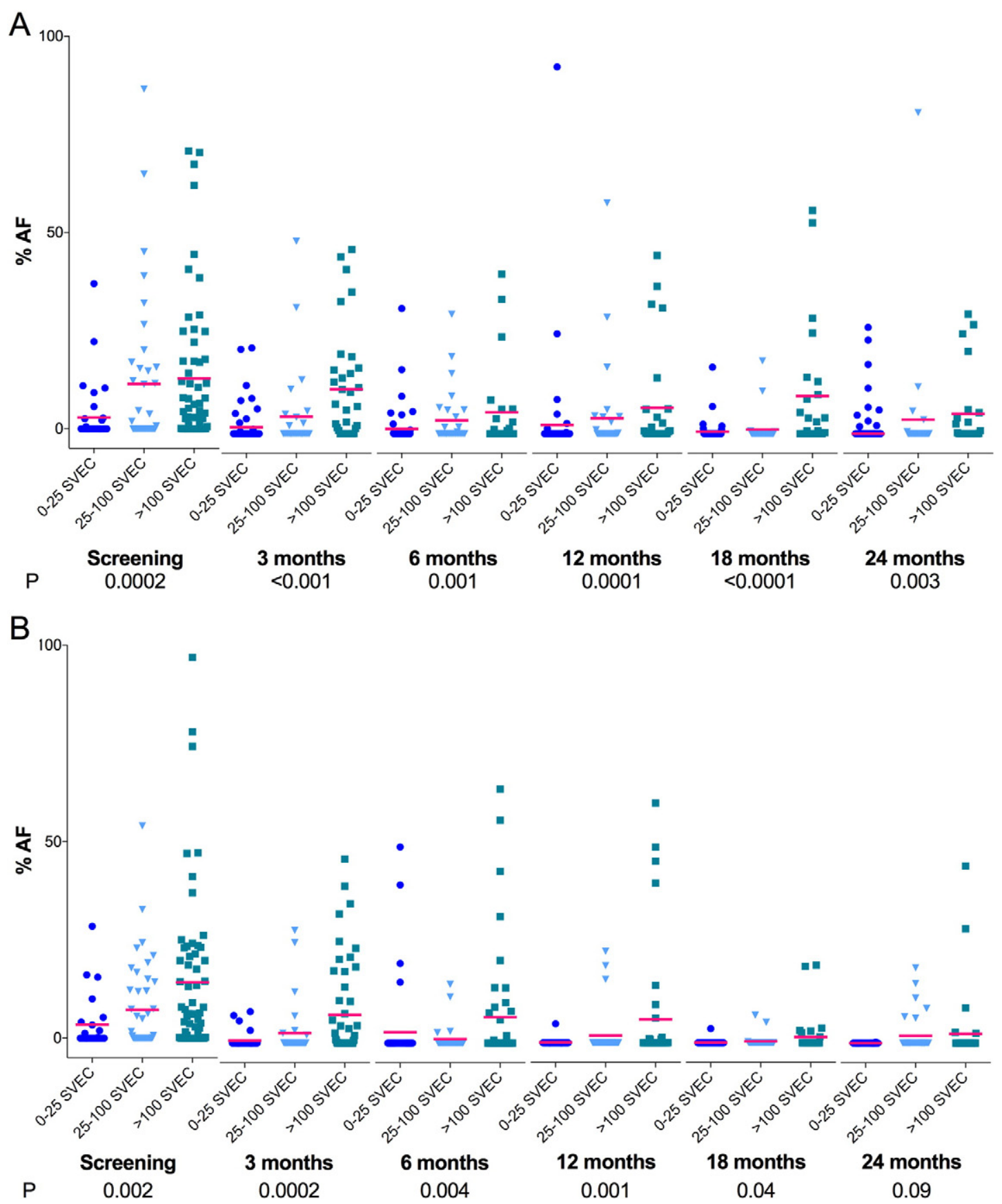

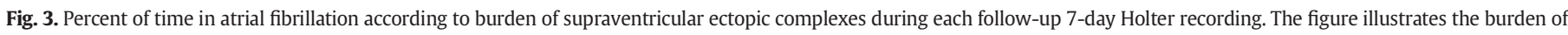

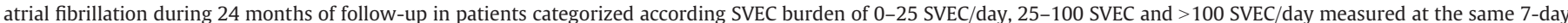

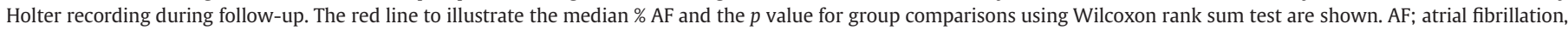
SVEC; supraventricular ectopic complexes.

24 months was very low in patients with the lowest SVEC burden whereas patients with low SVEC burden in the antiarrhythmic medication group had a higher AF burden. In this homogenous healthy study population with paroxysmal AF it is possible that most patients have pulmonary vein triggered $A F$ and these patients benefit from catheter ablation if the SVECs originating in the pulmonary veins are removed. However, after antiarrhythmic medication SVECs seems to be removed but the patients may still have SVEC originating in the pulmonary vein that triggers recurrent $\mathrm{AF}$ which could explain the higher $\mathrm{AF}$ burden in these patients. In the MANTRA PAF trial a large proportion of patients in the antiarrhythmic medication group underwent additional catheter ablation and thus the results tend to overestimate the effect of antiarrhythmic medication which further complicates the interpretation.
Nevertheless, our results indicate that patients with high SVEC burden represent an interesting patient population and taken together, these data suggest that new opportunities exist to further understand the role of SVEC burden in predicting outcome or changing the management of patients undergoing treatment of paroxysmal AF.

\subsection{Study limitations}

This study was performed as a post hoc analysis of a previous clinical trial with all the statistical limitations implied therein.

A major limitation of our results is that presence of AF excludes presence of SVEC and this could have affected our results. In addition, there are some limitations in the detection of SVEC using Holter 
monitoring that should be addressed. First, due to the short coupling interval to the preceding sinus beat SVECs can be concealed in the T wave causing difficulty in detecting SVEC. Furthermore, atrial depolarization results in a deflection on the surface-ECG of approximately $0.1 \mathrm{mV}$, which can easily be obscured by electrostatic noise arising from movement, skeletal muscle myopotentials and impaired skin contact during long-term recordings. Noise interference is the major cause of computer inaccuracies and is a major limitation in this study [22].

Patients were censored at the time of non-adherence to the randomized treatment. However, the non-adherence was more pronounced in patients assigned to antiarrhythmic medication where a large proportion of patients underwent supplementary catheter ablation during follow-up, which could cause selection bias.

\section{Conclusion}

Our results demonstrate that antiarrhythmic medication is superior to catheter ablation in suppressing SVEC burden during treatment of paroxysmal AF. After catheter ablation SVEC burden increased immediately post-procedural followed by a decrease during follow-up whereas after antiarrhythmic medication an early decrease was observed. Finally, lower SVEC burden was highly associated with lower AF burden during follow-up especially after CA.

\section{Funding}

This study was supported by Hans and Nora Buchard's Foundation, Aage and Gerda Hensch's Foundation and Jens Anker Andersen's Foundation.

\section{Conflicts of interest}

Christian Jons, MD CONSULTANT FEES/HONORARIA - Biotronik. Jens C. Nielsen, MD CONSULTANT FEES/HONORARIA - Biosense Webster,Biotronik.

No other conflicts of interest.

\section{Appendix A. Supplementary data}

Supplementary data to this article can be found online at http://dx. doi.org/10.1016/j.ijcard.2017.05.028.

\section{References}

[1] P. Kirchhof, S. Benussi, D. Kotecha, A. Ahlsson, D. Atar, B. Casadei, et al., 2016 ESC guidelines for the management of atrial fibrillation developed in collaboration with EACTS, Eur. Heart J. 37 (38) (2016 Oct 7) 2893-2962.

[2] F. Ouyang, R. Tilz, J. Chun, B. Schmidt, E. Wissner, T. Zerm, et al., Long-term results of catheter ablation in paroxysmal atrial fibrillation lessons from a 5-year follow-up, Circulation 122 (23) (2010 Dec 7) 2368-2377.

[3] A. Hakalahti, F. Biancari, J.C. Nielsen, M.J.P. Raatikainen, Radiofrequency ablation vs. antiarrhythmic drug therapy as first line treatment of symptomatic atrial fibrillation: systematic review and meta-analysis, Europace 17 (3) (Feb 26 2015) 370.
[4] M. Haïssaguerre, P. Jaïs, D.C. Shah, A. Takahashi, M. Hocini, G. Quiniou, et al., Spontaneous initiation of atrial fibrillation by ectopic beats originating in the pulmonary veins, N. Engl. J. Med. 339 (10) (1998) 659-666.

5] S.A. Chen, M.H. Hsieh, C.T. Tai, C.F. Tsai, V.S. Prakash, W.C. Yu, et al., Initiation of atrial fibrillation by ectopic beats originating from the pulmonary veins: electrophysiological characteristics, pharmacological responses, and effects of radiofrequency ablation, Circulation 100 (18) (1999 Nov 2) 1879-1886.

[6] S.M. Narayan, D. Kazi, D.E. Krummen, W.-J. Rappel, Repolarization and activation restitution near human pulmonary veins and atrial fibrillation initiation: a mechanism for the initiation of atrial fibrillation by premature beats, J. Am. Coll. Cardiol. 52 (15) (2008 Oktober) 1222-1230.

[7] C. Kolb, S. Nürnberger, G. Ndrepepa, B. Zrenner, A. Schömig, C. Schmitt, Modes of initiation of paroxysmal atrial fibrillation from analysis of spontaneously occurring episodes using a 12-lead Holter monitoring system, Am. J. Cardiol. 88 (8) (2001 Oktober) 853-857.

[8] H.-N. Pak, C. Hwang, H.E. Lim, J.W. Kim, H.S. Lee, Y.-H. Kim, Electroanatomic characteristics of atrial premature beats triggering atrial fibrillation in patients with persistent versus paroxysmal atrial fibrillation, J. Cardiovasc. Electrophysiol. 17 (8) (2006 Aug 1) 818-824.

[9] U.J.O. Gang, C.J. Nalliah, T.W. Lim, A. Thiagalingam, P. Kovoor, D.L. Ross, et al., Atrial ectopy predicts late recurrence of atrial fibrillation after pulmonary vein isolation, Circ. Arrhythm. Electrophysiol. 8 (3) (2015 Jun 1) 569-574.

[10] T. Yamane, T. Date, Y. Kanzaki, K. Inada, S. Matsuo, K. Shibayama, et al., Behavior of atrial ectopic beats before and after pulmonary vein isolation in patients with atrial fibrillation: a reduction in the number and arrhythmogenicity of ectopic firings, Heart Rhythm Off. J. Heart Rhythm Soc. 3 (12) (2006 Dec) 1421-1427.

[11] C. Alhede, A. Johannessen, U. Dixen, J.S. Jensen, P. Raatikainen, G. Hindricks, et al., Higher burden of supraventricular ectopic complexes early after catheter ablation for atrial fibrillation is associated with increased risk of recurrent atrial fibrillation, Europace 2016 (Dec 23 2016) http://dx.doi.org/10.1093/europace/euw329.

[12] P. Kirchhof, M. Engelen, M.R. Franz, M. Ribbing, K. Wasmer, G. Breithardt, et al., Electrophysiological effects of flecainide and sotalol in the human atrium during persistent atrial fibrillation, Basic Res. Cardiol. 100 (2) (Mar 1 2005) 112-121.

[13] J. Cosedis Nielsen, A. Johannessen, P. Raatikainen, G. Hindricks, H. Walfridsson, O. Kongstad, et al., Radiofrequency ablation as initial therapy in paroxysmal atrial fibrillation, N. Engl. J. Med. 367 (17) (2012) 1587-1595.

[14] C. Jons, P.S. Hansen, A. Johannessen, G. Hindricks, P. Raatikainen, O. Kongstad, et al., The medical antiarrhythmic treatment or radiofrequency ablation in paroxysmal atrial fibrillation (MANTRA-PAF) trial: clinical rationale, study design, and implementation, Europace 11 (7) (Jun 22 2009) 917.

[15] C. Varounis, N. Dagres, T. Maounis, D. Panagiotakos, D.V. Cokkinos, Atrial premature complexes and heart rate have prognostic significance in 1-month atrial fibrillation recurrence after electrical cardioversion, Europace 9 (8) (Aug 1 2007) 633-637.

[16] M. Takigawa, A. Takahashi, T. Kuwahara, K. Okubo, Y. Takahashi, Y. Watari, et al., Long-term outcome after catheter ablation of paroxysmal atrial fibrillation: impact of different atrial fibrillation foci, Int. J. Cardiol. 227 (Jan 15 2017) 407-412.

[17] S.A. Chen, C.T. Tai, C.F. Tsai, M.H. Hsieh, Y.A. Ding, M.S. Chang, Radiofrequency catheter ablation of atrial fibrillation initiated by pulmonary vein ectopic beats, J. Cardiovasc. Electrophysiol. 11 (2) (Feb 2000) 218-227.

[18] H. Calkins, K.H. Kuck, R. Cappato, J. Brugada, A.J. Camm, S.-A. Chen, et al., 2012 HRS/ EHRA/ECAS expert consensus statement on catheter and surgical ablation of atrial fibrillation: recommendations for patient selection, procedural techniques, patient management and follow-up, definitions, endpoints, and research trial design, Europace 14 (4) (Apr 1 2012) 528-606.

[19] H.S. Lim, C. Schultz, J. Dang, M. Alasady, D.H. Lau, A.G. Brooks, et al., Time course of inflammation, myocardial injury, and prothrombotic response after radiofrequency catheter ablation for atrial fibrillation, Circ. Arrhythm. Electrophysiol. 7 (1) (Feb 1 2014) 83-89.

[20] T.T. Issac, H. Dokainish, N.M. Lakkis, Role of inflammation in initiation and perpetuation of atrial fibrillation: a systematic review of the published data, J. Am. Coll. Cardiol. 50 (21) (Nov 20 2007) 2021-2028.

[21] J.E.P. Waktare, K. Hnatkova, S.M. Sopher, F.D. Murgatroyd, X. Guo, A.J. Camm, et al., The role of atrial ectopics in initiating paroxysmal atrial fibrillation, Eur. Heart J. 22 (4) (Feb 1 2001) 333-339.

[22] C. Members, A.H. Kadish, A.E. Buxton, H.L. Kennedy, B.P. Knight, J.W. Mason, et al., ACC/AHA clinical competence statement on electrocardiography and ambulatory electrocardiography, Circulation 104 (25) (Dec 18 2001) 3169-3178. 